\title{
Visual and Anatomical Outcomes Following Idiopathic Epiretinal Membrane and Internal Limiting Membrane Peeling
}

\author{
Gokhan Demir, Ali Demircan, Dilek Yasa, Husna Topcu, Erdem Eris, Gurkan Erdogan, Zeynep Alkin \\ University of Health Sciences Beyoglu Eye Training and Research Hospital, Istanbul, Turkey
}

\begin{abstract}
Objectives: The aim of this study was to evaluate the functional and anatomical outcomes and the rate of epiretinal membrane (ERM) recurrence in eyes that had undergone ERM-internal limiting membrane (ILM) peeling surgery at least 6 months earlier.

Methods: The records of $8 \mathrm{I}$ eyes of $8 \mathrm{I}$ patients who underwent pars plana vitrectomy (PPV) and ERM-ILM peeling with the diagnosis of idiopathic ERM between January 2015 and January 2016 in our clinic were retrospectively reviewed. Patients with at least 6 months of follow-up were included. The demographic details of the patients, duration of the followup period, use of endotamponade, and any complications were recorded. Best corrected visual acuity and central macular thickness (CMT) at baseline and at 6, I2, and 18 months were also recorded.

Results: A total of 8 leyes of 8 I patients, 45 (55.6\%) males and $36(44.4 \%)$ females, with a group mean age of $65.7 \pm 8.5$ years (range: $46-85$ years) were assessed. The mean preoperative visual acuity was $0.81 \pm 0.50$ logMAR (the logarithm of the minimum angle of resolution) ( 0.22 decimals). During the follow-up, the mean visual acuity at 6,12 , and 24 months was $0.61 \pm 0.37 \log$ MAR ( 0.33 decimals), $0.61 \pm 0.38 \log$ MAR $(0.33$ decimals), and $0.55 \pm 0.33$ logMAR $(0.36$ decimals), respectively. The visual acuity was significantly better at 6,12, and 24 months compared with the baseline measurement

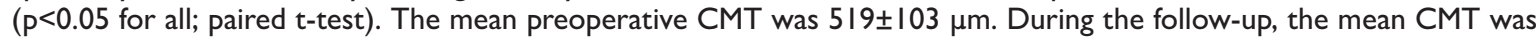
$302 \pm 89 \mu \mathrm{m}, 287 \pm 80 \mu \mathrm{m}$, and $273 \pm 82 \mu \mathrm{m}$ at 6,12 , and 24 months, respectively. Compared with the baseline, a statistically significant difference was observed at 6, 12 , and 24 months.

Conclusion: PPV with membranectomy and ILM peeling is a safe and effective method to treat idiopathic ERM. It led to a functional improvement and a reduction in macular thickness in most of the patients diagnosed with ERM. The rate of recurrence of ERM and the need for repeat ERM surgery was lower in eyes where the ILM was removed with the ERM than the expected rate for when ERM peeling is performed alone.
\end{abstract}

Keywords: Epiretinal membrane, internal limiting membrane peeling, pars plana vitrectomy.

\section{Introduction}

An epiretinal membrane (ERM) is a common cause of visual impairment, with a reported prevalence of $7 \%$ to $11.8 \% .1 \mathrm{lt}$ is a condition that mostly affects people over the age of 50 years (2). In the majority of cases, the membrane is usually asymptomatic, but it may become symptomatic if the macu- lar or perimacular areas are involved (3). The most common symptoms include decreased visual acuity and metamorphopsia, although micropsia and monocular diplopia have also been reported (4). ERM can be idiopathic or secondary to many conditions, such as posterior vitreous detachment, ocular inflammatory diseases, retinal vascular occlusions, intraocular tumors, and retinal dystrophies (5). 
Pars plana vitrectomy (PPV) and membrane peeling, first described by Machemer (6) in 1978, have become the established surgical treatment for ERM. The surgical removal of an ERM has been reported to be a safe procedure with low rates of complications and high rates of visual recovery (7). Idiopathic ERM recurrence is seen in approximately $10 \%$ of cases without ILM peeling, and reoperation is required in approximately $3 \%$ of cases (8). Many vitreoretinal surgeons favor ILM peeling during ERM surgery, because it facilitates retinal striae resolution and reduces the recurrence rate of ERM (9). It has previously been demonstrated that the combination of ERM peeling and internal limiting membrane (ILM) peeling reduced the rate of recurrence to less than $3 \%$.

The objective of the present study was to evaluate the functional and anatomical outcome and the rate of ERM recurrence in eyes that had undergone ERM-ILM peeling surgery at least 6 months earlier.

\section{Methods}

A total of $8 \mathrm{I}$ eyes of $8 \mathrm{I}$ patients who underwent PPV and ERM-ILM peeling with the diagnosis of idiopathic ERM between January 2015 and January 2016 in the clinic were retrospectively reviewed. The diagnosis of ERM was made clinically with a fundus examination, fundus photography, and spectral-domain optical coherence tomography (SD-OCT) (Spectralis; Heidelberg Engineering, AG, Heidelberg, Germany). Patients with at least 6 months of follow-up were included. Exclusion criteria were ERM secondary to ocular diseases affecting the retina, such as diabetic retinopathy, venous occlusion, retinal detachment, uveitis, and age-related macular degeneration; ocular trauma; myopia of more than 6 diopters or an axial length greater than $26 \mathrm{~mm}$; full-thickness or lamellar macular hole; opaque optical media that significantly affect vision or disrupt OCT imaging; or a history of prior intraocular surgery within 6 months.

Initially, a full ophthalmic examination, including measurement of best corrected visual acuity (BCVA) with a Snellen chart, anterior and posterior segment examination with slit-lamp biomicroscopy, color fundus photography, fluorescein angiography (HRA-2; Heidelberg Engineering, AG, Heidelberg, Germany), and SD-OCT imaging were performed. Demographic details of the patients, the duration of the follow-up period, use of an endotamponade, and any complications were recorded. The BCVA and central macular thickness (CMT) at baseline and at 6, 12, and 24 months were also recorded.

\section{Surgical Technique}

The surgical procedure involved a 3-port vitrectomy using the Constellation system (Alcon, Hünenberg, Switzerland) using $23-G$, valved trocars and a widefield viewing system.
After removal of the vitreous gel and posterior hyaloid, ERM peeling was performed using 23-G forceps. After removal of the ERM with the assistance of tryphan blue staining, the ILM was stained with brilliant blue solution. The ILM was peeled from an area within 2 to 3 disc diameters from the fovea using 23-G forceps. In order to minimize the damage to the papillomacular bundle, the ERM and ILM peeling were initiated at the temporal region around the fovea. Following fluid-air exchange, an intravitreal gas injection was performed in some cases when necessary.

\section{Statistical Analysis}

IBM SPSS Statistics for Windows, Version 22.0 IBM Corp., Armonk, NY, USA) was used for the statistical analysis. The normality of distribution of the quantitative data was assessed using the Kolmogorov-Smirnov test, Shapiro-Wilk test, and variability coefficients. Parametric methods were used in the analysis of variables with normal distribution. A paired-samples T-test was used for comparisons. For the statistical analysis, all visual acuity values were converted to the logarithm of the minimum angle of resolution (logMAR). Significance was established at $p<0.05$.

\section{Results}

In all, 81 eyes of 81 patients, $45(55.6 \%)$ of whom were males and $36(44.4 \%)$ were females, with a group mean age of $65.7 \pm 8.5$ years (range: $46-85$ years) were assessed retrospectively. Table I summarizes the demographic characteristics of the patients.

All of the patients were followed up for at least 6 months: 67 eyes were followed up for 12 months, and 48 eyes for at least 24 months. The mean length of the follow-up period was 18.3 \pm 10 months (range: 6-27 months).

Table I. The demographic characteristics of the patients

\begin{tabular}{lc} 
& $\mathbf{n}=\mathbf{8} \mathbf{I}$ \\
\hline Age (years) & $65.7 \pm 8.5$ \\
Gender & \\
Male & $45(55.6)$ \\
Female & $36(44.4)$ \\
\hline
\end{tabular}

Table 2. The change in mean central macular thickness

\section{CMT}

p

\begin{tabular}{lrl}
\hline Baseline & $519 \pm 103$ & \\
At 6 months & $302 \pm 89$ & $<0.05$ \\
At 12 months & $287 \pm 80$ & $<0.05$ \\
At 24 months & $273 \pm 82$ & $<0.05$
\end{tabular}

CMT: Central macular thickness. 


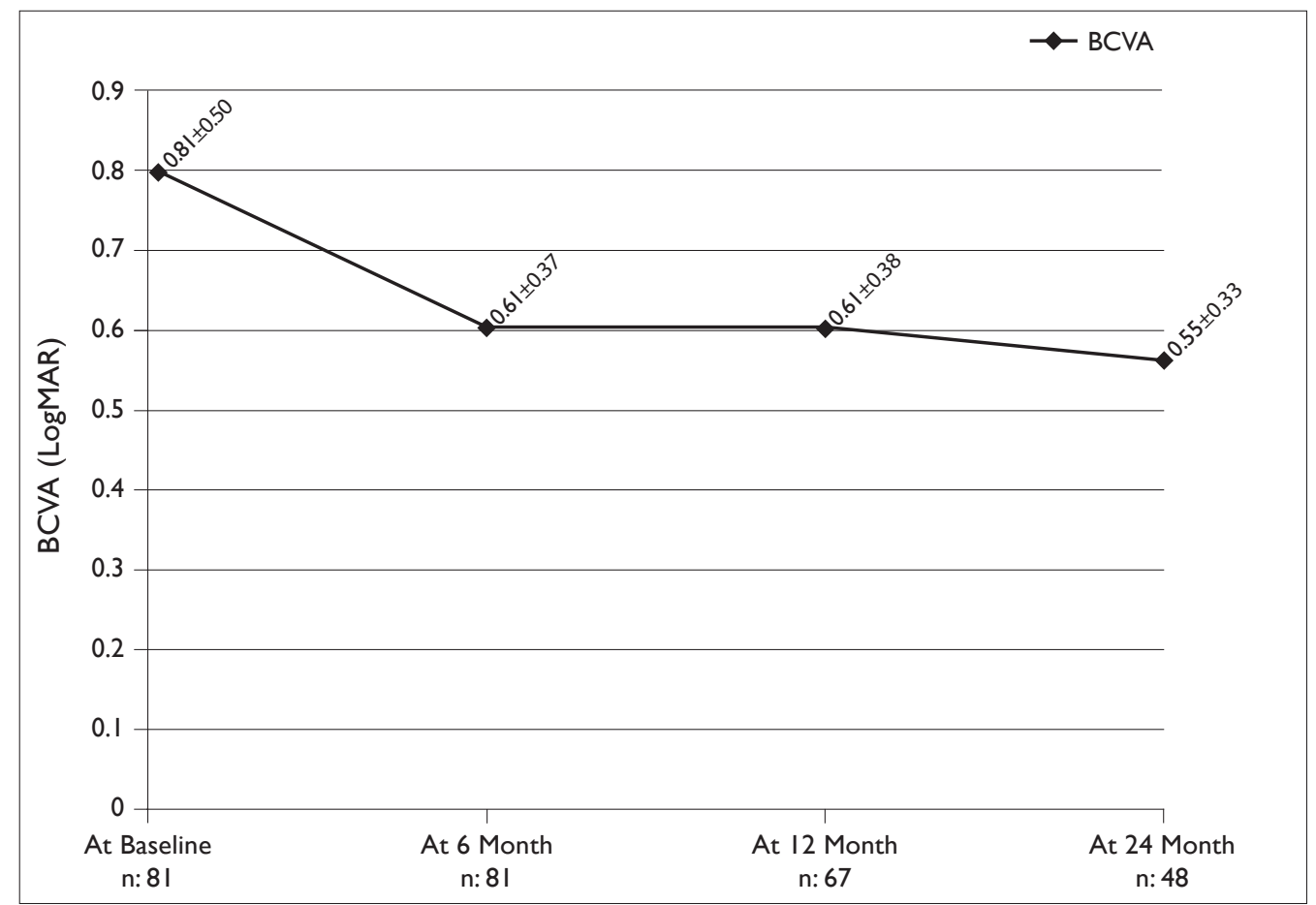

Figure I. The change in mean logMAR best corrected visual acuity. BCVA: Best corrected visual acuity.

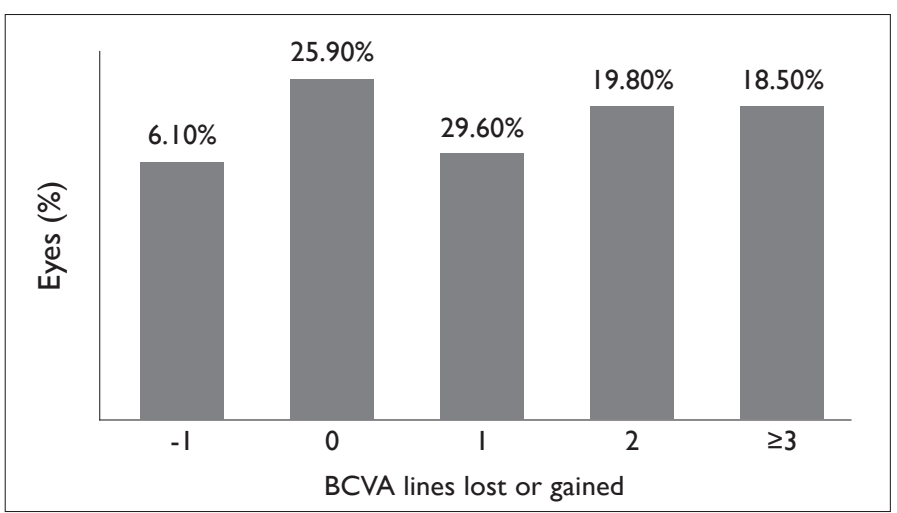

Figure 2. Percentage of eyes that lost or gained lines of BCVA at the last follow-up visit.

BCVA: Best corrected visual acuity.

The mean preoperative visual acuity was $0.81 \pm 0.50 \mathrm{log}$ MAR ( 0.22 decimals). During the follow-up, the mean visual acuity at 6,12 , and 24 months was $0.6 I \pm 0.37 \log$ MAR $(0.33$ decimals), $0.6 I \pm 0.38$ logMAR (0.33 decimals), and $0.55 \pm 0.33$ logMAR (0.36 decimals), respectively. The visual acuity was significantly better at 6,12 , and 24 months compared with the baseline measurement ( $p<0.05$ for all; paired $t$-test). Figure I illustrates the changes in mean logMAR BCVA. At the end of the follow-up period, BCVA increased I line in 24 eyes (29.6\%), 2 lines in 16 eyes (19.8\%), $\geq 3$ lines in 15 eyes ( $18.5 \%$ ), remained unchanged in 21 eyes $(25.9 \%)$, and decreased in 5 eyes (6.2\%). Figure 2 shows the lines gained and lost.
The mean preoperative CMT was $519 \pm 103 \mu \mathrm{m}$. During the follow-up, the mean CMT was $302 \pm 89 \mu \mathrm{m}, 287 \pm 80 \mu \mathrm{m}$, and $273 \pm 82 \mu \mathrm{m}$ at 6,12 , and 24 months, respectively. Compared with the baseline, a statistically significant difference was observed at 6,12 , and 24 months. The changes in mean CMT can be seen in Table 2 .

Sulfur hexafluoride was used as an endotamponade in 9 (II.1\%) patients, octafluoropropane in $3(3.7 \%)$ patients, and air in 9 (II.I) patients. The remaining 60 (74.I\%) patients were closed with fluid. In up to 2 years of follow-up, no ERM recurrence was seen in any patient; however cataract progressed in 9 patients and phacoemulsification surgery was performed on these patients.

No intraoperative or postoperative complications, such as retinal detachment, vitreous hemorrhage, persistently elevated IOP, or endophthalmitis, were observed.

\section{Discussion}

An ERM develops at the vitreomacular interface and is determined by the proliferation of a different type of cells, which produce collagen and migrate onto the ILM. These cells gradually form a transparent hypocellular avascular layer, and like all scar tissue, tighten to create tension on the retina, which may bulge and pucker, or even cause swelling or macular edema (I0). The ILM is the basement membrane of the Muller cells and can act as a scaffold for cellular proliferation in the pathophysiology of disorders affecting the vit- 
reomacular interface (I0). Until I990, the ILM was considered an integral part of the retina, and vitreoretinal surgeons did not think that it could be removed without causing damage to vision. Histological examination of the removed ERM has shown that in $40 \%$ to $60 \%$ of patients, the ILM and ERM are so adherent they are often removed together at the same time, thereby confirming the hypothesis that these 2 membranes are strictly linked in causing epiretinal puckering $(\mathrm{II}, \mathrm{I2})$. The atraumatic removal of the macular ILM has been proposed to treat various forms of tractional maculopathy, in particular for macular pucker. In the last decade, the removal of the ILM has become routine practice in ERM surgery, and has had good anatomical results. However, many recent studies have reported that ILM peeling is a procedure that can cause immediate traumatic effects and progressive modification of the underlying inner retinal layers. Moreover, it is unclear whether ILM peeling is helpful to improve vision after surgery for ERM (10).

The results of this 24-month follow-up study demonstrated that PPV and ERM-ILM peeling was effective in restoring visual acuity in patients and decreasing the recurrence of ERM. The ERM recurrence rates reported in the literature range between $5 \%$ and $12 \%$, and the follow-up of those studies was generally up to 12 months and rarely beyond $(13,14)$. In the present study, no ERM recurrence was seen in any patient during the 2-year follow-up period.

There are several studies that compared ERM peeling with ILM peeling and without ILM peeling in the literature. Tranos et al.(I5) reported that the mean change in distance BCVA at 12 months was $0.30 \pm 0.24 \log M A R$ (I5 ETDRS letters) in the peeling group and $0.31 \pm 0.23$ logMAR (I4 ETDRS letters) in the non-peeling group, a change that was not statistically significant $(p=0.84)$. No statistically significant differences were observed when comparing the changes in distance BCVA, changes in metamorphopsia (Amsler grid), and changes in central retinal thickness between the groups at any of the time points. Oh et al. (16) reviewed the medical records of 43 patients with an idiopathic ERM who underwent vitrectomy and ERM-ILM removal between July 2007 and April 2010. There was no recurrence of an ERM, as in our study. Kwok et al. (13) reported an ERM recurrence rate of $17.6 \%$ for surgery with no membrane peeling compared with a recurrence rate of $0 \%$ in the ILM peeling group. Shimada et al. (14) found similar results of a higher recurrence rate in the non-ILM-peeling group. Recently, in a study performed by Schechet et al. (17), I 40 eyes (55.8\%) did not have an ILM peel (non-ILM group), and I I I eyes (44.2\%) did have an ILM peel (ILM group). There was no significant difference in the final BCVA $(p=0.18)$ or total change of BCVA $(p=0.48)$. Some degree of ERM recurrence was detected by slit-lamp biomicroscopy in 2 eyes (1.8\%) of the ILM group and in 32 eyes $(22.9 \%)$ of the non-ILM group $(p \leq 0.000 \mathrm{I})$. A retrospective study of 39 eyes operated on for idiopathic ERM was conducted. In Group A (without ILM peeling), the mean preoperative BCVA was 0.48 logMAR ( 0.3 decimals), whereas the mean postoperative BCVA was 0.37 logMAR (0.4 decimals). In Group B (with ILM peeling), the mean preoperative BCVA was 0.58 logMAR ( 0.25 decimals), whereas the mean postoperative BCVA was 0.31 logMAR ( 0.5 decimals). No statistically significant difference was observed between Group A and Group B regarding preoperative or postoperative BCVA. OCT measurement of postoperative foveal thickness revealed a significant decrease in thickness in both groups. They reported that ILM peeling did not affect the functional outcome of idiopathic ERM removal (I8).

The current study has some limitations. First, this is a retrospective study. Additionally, this study had a small sample size, there was no comparison group, and the follow-up period was short.

In conclusion, PPV with membranectomy and ILM peeling is a safe and effective method to treat idiopathic ERM. It led to a functional improvement and a reduction in macular thickness in most of the patients. The rate of recurrence of ERM and need for repeat ERM surgery was lower in eyes where the ILM was removed with the ERM than the expected rate for when ERM peeling is performed alone

\section{Disclosures}

Peer-review: Externally peer-reviewed.

Conflict of Interest: None declared.

Authorship Contributions: Involved in design and conduct of the study (GD, ZTA, ALD); preparation and review of the study (GD, ZTA, GE); data collection (HT, DY, ALD); and statistical analysis (GD, EE, ALD).

\section{References}

I. Mitchell P, Smith W, Chey T, Wang JJ, Chang A. Prevalence and associations of epiretinal membranes. The Blue Mountains Eye Study, Australia. Ophthalmology 1997; 104: 1033-40. [CrossRef]

2. Folk JC, Adelman RA, Flaxel CJ, Hyman L, Pulido JS, Olsen TW. Idiopathic Epiretinal Membrane and Vitreomacular Traction Preferred Practice Pattern(®) Guidelines. Ophthalmology 2016; 123:PI 52-8I. [CrossRef]

3. Arimura E, Matsumoto C, Okuyama S, Takada S, Hashimoto S, Shimomura Y. Retinal contraction and metamorphopsia scores in eyes with idiopathic epiretinal membrane. Invest Ophthalmol Vis Sci 2005;46:296I-6. [CrossRef]

4. Margherio RR, Cox MS Jr, Trese MT, Murphy PL, Johnson J, Minor LA. Removal of epimacular membranes. Ophthalmology 1985;92:1075-83. [CrossRef]

5. Bu SC, Kuijer R, Li XR, Hooymans JM, Los LI. Idiopathic epiretinal membrane. Retina 20I4;34:23।7-35. [CrossRef]

6. Machemer R. The surgical removal of epiretinal macular mem- 
branes (macular puckers) (author's transl). Klin Monbl Augenheilkd 1978; I73:36-42.

7. Sandali O, El Sanharawi M, Lecuen N, Barale PO, Bonnel S, Basli $\mathrm{E}$, et al. 25-, 23-, and 20-gauge vitrectomy in epiretinal membrane surgery: a comparative study of 553 cases. Graefes Arch Clin Exp Ophthalmol 201 I;249:181 I-9. [CrossRef]

8. Sandali O, El Sanharawi M, Basli E, Bonnel S, Lecuen N, Barale $\mathrm{PO}$, et al. Epiretinal membrane recurrence: incidence, characteristics, evolution, and preventive and risk factors. Retina 20।3;33:2032-8. [CrossRef]

9. Sorcinelli R. Surgical management of epiretinal membrane with indocyanine-green-assisted peeling. Ophthalmologica 2003;2 17:107-10. [CrossRef]

10. Semeraro F, Morescalchi F, Duse S, Gambicorti E, Russo A, Costagliola C. Current Trends about Inner Limiting Membrane Peeling in Surgery for Epiretinal Membranes. J Ophthalmol 2015;2015:671905. [CrossRef]

I I. Kuhn F. Point: to peel or not to peel, that is the question. Ophthalmology 2002; 109:9-II. [CrossRef]

12. Gibran SK, Flemming B, Stappler T, Pearce I, Groenewald $\mathrm{C}$, Heimann $\mathrm{H}$, et al. Peel and peel again. $\mathrm{Br} J$ Ophthalmol
2008;92:373-7. [CrossRef]

13. Kwok AKh, Lai TY, Yuen KS. Epiretinal membrane surgery with or without internal limiting membrane peeling. Clin Exp Ophthalmol 2005;33:379-85. [CrossRef]

14. Shimada H, Nakashizuka H, Hattori T, Mori R, Mizutani Y, Yuzawa $M$. Double staining with brilliant blue $G$ and double peeling for epiretinal membranes. Ophthalmology 2009; I 16:1370-6.

15. Tranos P, Koukoula S, Charteris DG, Perganda G, Vakalis A, Asteriadis $S$, et al. The role of internal limiting membrane peeling in epiretinal membrane surgery: a randomised controlled trial. $\mathrm{Br}$ J Ophthalmol 2017;101:719-24. [CrossRef]

16. Oh HN, Lee JE, Kim HW, Yun IH. Clinical outcomes of double staining and additional ILM peeling during ERM surgery. Korean J Ophthalmol 2013;27:256-60. [CrossRef]

17. Schechet SA, DeVience E, Thompson JT. The effect of internal limiting membrane peeling on idiopathic epiretinal membrane surgery, with a review of the literature. Retina 2017;37:873-80.

18. Pournaras CJ, Emarah A, Petropoulos IK. Idiopathic macular epiretinal membrane surgery and ILM peeling: anatomical and functional outcomes. Semin Ophthalmol 201 I;26:42-6. [CrossRef] 